\title{
An Uncommon Etiology for Pleural Effusion with Rib Lesion in a 78-year-old Woman: A Case Report
}

De-an Qin ( $\nabla$ qindean.student@sina.com )

Shanxi Provincial Peoples Hospital https://orcid.org/0000-0002-3025-2706

\section{Xiao-qiang Ren}

Shanxi Provincial Peoples Hospital

Jie Jiang

Shanxi Armed Police Hospital

\section{Yun-peng Wang}

Shanxi Provincial Peoples Hospital

\section{Case report}

Keywords: Pleural effusion, Rib lesion, Multiple myeloma, Diagnosis

Posted Date: October 15th, 2020

DOI: https://doi.org/10.21203/rs.3.rs-91617/v1

License: (c) (1) This work is licensed under a Creative Commons Attribution 4.0 International License. Read Full License 


\section{Abstract}

Background: Pleural effusion with rib lesion is generally considered as metastasis of thoracic carcinoma or rib tuberculosis and tuberculous pleuritis. Myelomatous pleural effusion with rib lesion is rare and poses a great diagnostic challenge. We described an easily-misdiagnosed extrapulmonary disease with concomitant pulmonary and rib manifestations, bilateral pleural effusion with lytic rib lesion in multiple myeloma, in order to raise awareness of this disease and avoid misdiagnose.

Case presentation: A 78-year-old woman was referred to the thoracic department with chest distress, breathlessness, poor appetite and weight loss for 3 months. Chest computed tomography demonstrated bilateral pleural effusion and lytic lesion on the left 5th rib. Whole-body bone scan showed the intense uptake lesions on the sixth, seventh vertebrae and a central defect with the peripheral uptake of the "donut"-like change sign on the left 5th rib. Percutaneous CT-guided core biopsy for the rib lesion was performed and the diagnosis was multiple myeloma. Combined chemotherapy and supportive treatment were prescribed. She did not respond to the therapy and died 3 months later due to pleural cavity infection and cardiopulmonary failure.

Conclusion: Some uncommon extrapulmonary diseases with concomitant pulmonary and rib manifestations should be reminded in order to avoid misdiagnose. Extramedullary multiple myeloma can affect any tissue and is an aggressive entity with poor prognosis. Percutaneous CT-guided core biopsy for the rib lesion is of higher positive rate than pleural fluid cytology and more minimally invasive than pleural biopsy via thoracoscope or rib excision biopsy.

\section{Background}

Pleural effusion with rib lesion is generally considered as metastasis of thoracic carcinoma or rib tuberculosis and tuberculous pleuritis. In patients not receiving active immunosuppressive therapy, some underlying hematologic diseases can inflict the chest(1). Multiple myeloma (MM) is the second most common hematological malignancy affecting mainly the elderly. Extramedullary multiple myeloma (EM) can affect any area of tissue including the breast, bone, lung, or pleura and is an aggressive, mostly resistant entity with poor prognosis. The incidence of EM varies largely among studies, possibly due to different imaging technique used (2). Myelomatous pleural effusion (MPE) is uncommon with $6 \%$ of patients with MM (3). MPE with rib lesion is rare and poses a great diagnostic challenge. We reported a unique case of symptomatic bilateral pleural effusion with lytic rib lesion in MM. Then percutaneous CTguided core biopsy for the rib lesion was performed and the diagnosis was established. This method was of higher positive rate than pleural fluid cytology and more minimally invasive than pleural biopsy via thoracoscope or rib excision biopsy.

\section{Case Presentation}


A 78-year-old woman with a history of hypertension was referred to the thoracic department with chest distress, breathlessness, poor appetite and weight loss for 3 months. She had family history of lung cancer for her brother. Physical examination revealed tachypnea and weak breath sounds on both lungs. There was palpable, tender, swollen 5th rib anteriorly at the level of left nipple. Chest computed tomography (CT) demonstrated bilateral pleural effusion and lytic lesion on the left 5 th rib whose cortex was destroyed (Fig. 1a). Whole-body bone scan was performed 3 hours after the injection of $20 \mathrm{mCi}$ $99 \mathrm{mTc}-\mathrm{MDP}$ and showed the intense uptake lesions on the sixth, seventh vertebrae and a central defect with the peripheral uptake of the "donut"-like change sign on the left 5 th rib, suggesting multiple, metastatic lesions (Fig. 1b). Laboratory findings revealed normal erythrocyte sedimentation rate, Creactive protein, blood cell analysis, electrolate, tumor markers, procalcitonin, TB-PCR, globulin, A/G ratio, $\lg A$ and $\lg \mathrm{G}$. The total protein was $54 \mathrm{~g} / \mathrm{L}$ (normal $58-80 \mathrm{~g} / \mathrm{L}$ ), albumin $30 \mathrm{~g} / \mathrm{L}$ (normal 38-60 g/L), serum creatinine $158 \mu \mathrm{mol} / \mathrm{L}$ (normal 44-88 $\mu \mathrm{mol} / \mathrm{L}$ ), $\beta 2$ microglobulin $6.92 \mathrm{mg} / \mathrm{L}$ (normal 0.8-3.0 mg/L) and $\mathrm{lgM} 0.36 \mathrm{~g} / \mathrm{L}$ (normal $0.63-2.77 \mathrm{~g} / \mathrm{L}$ ). Bence-Jones protein in urine and serum protein electrophoresis was negative. Thoracentesis revealed hemorrhagic effusion (positive Rivalta test and red blood cells). A pleural catheter was subsequently inserted into left pleural cavity. Pleural fluid tests for pyogenic bacteria

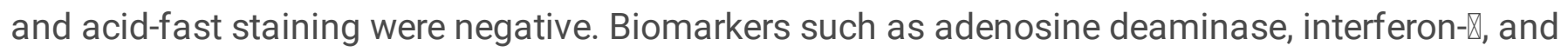
interleukin-27 were negative. Repetitive cytological examination found scattered cells with heterogeneous nuclear which were difficult to distinguish with adenocarcinoma. Bronchoscopy showed no endobronchial pathology. So, percutaneous CT-guided core biopsy using a 13-gauge core needle under local anesthesia was performed for the left 5 th rib lesion (Fig. 1c). Puncture pathology for rib lesion revealed abundant immature plasma cells (Fig. 1d). The immunohistochemistry of CD19, CD38 and CD138 were positive. A diagnosis of multiple myeloma (MM) was made. Subsequent bone marrow aspiration, immunofixation electrophoresis and cytogenetics confirmed as $\lambda$ type, ISS- $\mathbb{\text { stage and }}$ $46, X X[20]$. The surgery for rib resection was halted considering the poor prognosis and the patient underwent combined chemotherapy (bortezomib and dexamethasone) and supportive treatment. She did not respond to the therapy and died 3 months later due to pleural cavity infection and cardiopulmonary failure.

\section{Discussion And Conclusions}

Concomitant pulmonary and rib lesions usually are considered as metastases. Some uncommon extrapulmonary diseases with concomitant pulmonary and rib manifestations should be reminded in order to avoid misdiagnose. Lymphoma is the most common type to involve the chest wall and ribs and accounts for about $0.3-1.0 \%$ of extranodal lymphoma. It often appears as a large extraosseous softtissue mass, with relative preservation of the underlying rib cortex. Up to $10 \%$ of malignant pleural effusions are due to lymphoma(4). Multiple myeloma (MM) is the second most common hematological malignancy affecting mainly the elderly. The median age at presentation is approximately 70 years. The clonal plasma cells infiltration of bone marrow and presence of monoclonal immunoglobulin in serum and/or urine are its characteristics. Extramedullary multiple myeloma (EM) can affect any area of tissue including the breast, bone, lung, or pleura and is an aggressive, mostly resistant entity with poor 
prognosis. Myelomatous pleural effusion (MPE) is uncommon with $6 \%$ of patients with MM (3). It is usually related to secondary causes such as congestive heart failure, chronic renal failure, pulmonary infection, serious hypoalbuminemia and thrombosis. MPE with rib lesion is rarer and poses a great diagnostic challenge in need of better strategic approaches (5). Laboratory findings may reveal abnormal globulin, IgA, IgG, A/G ratio, or Bence-Jones protein; however, these are often insensitive and lagging like this case. Diagnostic procedures for MPE have been pleural fluid cytology or pleural biopsy via thoracoscope, as well as bone marrow biopsy. Cytological examination for malignant plasma cells in the pleural fluid has a limited diagnostic sensitivity. Despite guidance from NICE which positioned wholebody MRI as the first-line diagnostic imaging test for suspected myeloma (6), it is currently performed at only a few centers due to limited MRI capacity(7). $99 \mathrm{~m}$-Tc MDP bone scan is a sensitive but insufficient method for detecting bone lesions in $\mathrm{MM}(8)$ and may show the "donut"-like change due to the lytic center. Percutaneous CT-guided core biopsy for the rib lesion was of higher positive rate than pleural fluid cytology.

Rib destruction has many etiologies and is more likely to be caused by metastatic rather than primary bone tumors. Metastatic rib lesions commonly include the lung, breast, thyroid and kidney. Specific radiological findings, such as ground-glass patterns in fibrous dysplasia, trabecular patterns in hemangioma, calcification in osteochondroma and fluid levels in aneurysmal bone cyst, usually are benign bone tumors(9). Solitary plasmacytoma of the rib should be considered when confronted with a lytic rib tumor. It is often resectable with good prognosis (10). On the contrary, MPE with rib lesion is characterized by an aggressive clinical course with a low possibility of survival exceeding 6 months and the surgery is contraindicated (11).Traditional chemotherapy is less effective in MPE than in MM without PE. Researchers must develop more effective and durable treatments for MM patients with PE.

Some uncommon extrapulmonary diseases with concomitant pulmonary and rib manifestations should be reminded in order to avoid misdiagnose. Extramedullary multiple myeloma can affect any area of tissue and is an aggressive entity with poor prognosis. Percutaneous CT-guided core biopsy for the rib lesion is of higher positive rate than pleural fluid cytology and more minimally invasive than pleural biopsy via thoracoscope or rib excision biopsy.

\section{Abbreviations}

Pleural effusion (PE)

Multiple myeloma (MM)

Myelomatous pleural effusion (MPE)

Extramedullary multiple myeloma (EM)

Computed tomography (CT) 


\section{Declarations}

\section{Ethics approval and consent to participate:}

We have had the ethics approval and consent of the Shanxi Medical University ethics committee.

\section{Consent for publication:}

Written consent to publish this information had been obtained from the patient for publication of this case report and accompanying images. A copy of the written consent is available for review by the Editors-in-Chief of this journal.

\section{Availability of data and materials:}

The datasets generated and/or analysed during the current study are not publicly available till publication but are available from the corresponding author on reasonable request.

\section{Competing interests:}

The authors declare that they have no competing interests.

\section{Funding:}

There is no funding for the research.

\section{Authors' contributions:}

XQ R, YP W, and J J analyzed and interpreted the patient's data and the medical treatment. DA Q performed the biopsy procedure of the rib lesion, and was a major contributor in writing the manuscript. All authors read and approved the final manuscript.

\section{Acknowledgements:}

Not applicable.

\section{References}

1. Franquet T, Rosado-de-Christenson ML, Marchiori E, Abbott GF, Martinez-Jimenez S, Lopez L. Uncommon thoracic manifestations from extrapulmonary tumors: Computed tomography evaluation - Pictorial review. Respir Med. [Journal Article; Review]. 2020 2020-07-01;168:105986.

2. Sevcikova S, Minarik J, Stork M, Jelinek T, Pour L, Hajek R. Extramedullary disease in multiple myeloma - controversies and future directions. BLOOD REV. 2019 2019-01-01;36:32-9.

3. JIANG A, YANG Y, GAO X, LU H. Bilateral pleural effusion as an initial manifestation of multiple myeloma: A case report and literature review. EXP THER MED. 2015 2015-01-01;9(3):1040-2. 
4. Iwasa Y, Okada A, Takenaka H, Takahashi T, Koguchi N, Katayama K, et al. Primary Malignant Lymphoma Originating from the Chest Wall without Preceding Pleural Disease. Internal medicine (Tokyo, Japan). 2017 2017-01-01;56(6):681-6.

5. Sharma D. A Rare Case of Multiple Myeloma Presenting As Lytic Lesion of the Rib. JOURNAL OF CLINICAL AND DIAGNOSTIC RESEARCH. 2016.

6. Pratt G, Morris TC. Review of the NICE guidelines for multiple myeloma. INT J LAB HEMATOL. 2017 2017-02-01;39(1):3-13.

7. Barwick T, Bretsztajn L, Wallitt $K$, Amiras D, Rockall A, Messiou C. Imaging in myeloma with focus on advanced imaging techniques. The British journal of radiology. 2019 2019-0101;92(1095):20180768.

8. Vakili SM, Sedaghat S. Is $99 \mathrm{~m}$ Tc-methylene diphosphonate bone scintigraphy a sensitive method for detecting bone lesions in multiple myeloma? Caspian J Intern Med. [Journal Article]. 2018 201803-01;9(2):140-3.

9. Levine BD, Motamedi K, Chow K, Gold RH, Seeger LL. CT of rib lesions. AJR Am J Roentgenol. [Journal Article; Review]. 2009 2009-07-01;193(1):5-13.

10. Jia R, Xue L, Liang H, Gao K, Li J, Zhang Z. Surgery combined with radiotherapy for the treatment of solitary plasmacytoma of the rib: a case report and review of the literature. J CARDIOTHORAC SURG. 2015 2015-01-01;10(1):125.

11. Zhong $\mathrm{Y}$, Zhang J, Wang H. Myelomatous pleural effusion involvement in 23 patients with multiple myeloma: A single-center clinical analysis. THORAC CANCER. 2015 2015-01-01;6(3):359-62.

\section{Figures}




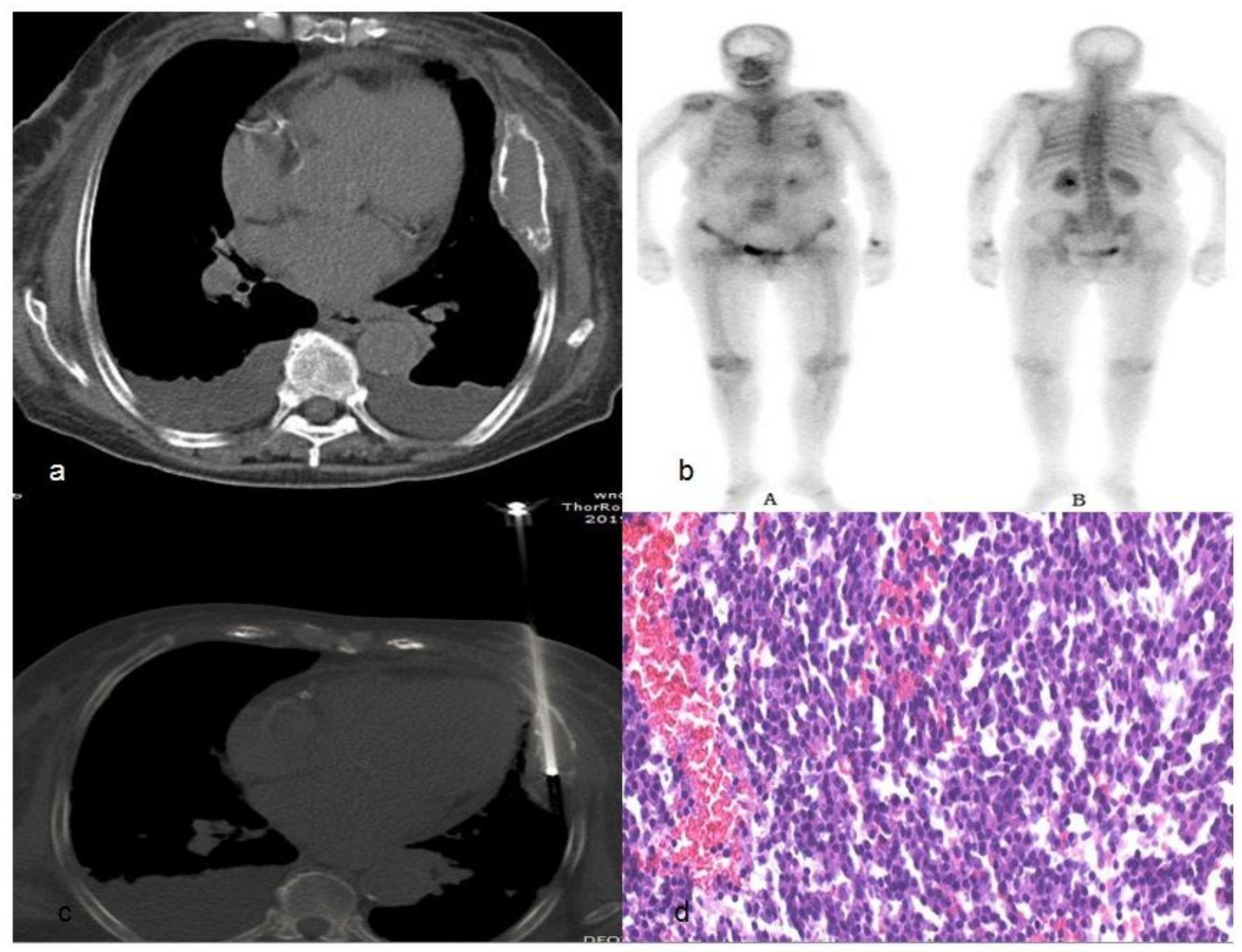

\section{Figure 1}

The imagings for a 78-year-old woman. a, chest CT showed bilateral pleural effusion and lytic left 5th rib lesion. b, whole-body bone scan showed the intense uptake lesions on the sixth, seventh vertebrae and a central defect with the peripheral uptake of the "donut"-like change sign on the left 5th rib. c, percutaneous CT-guided core biopsy of the left 5th rib lesion. $d$, puncture pathology for rib lesion revealing abundant immature plasma cells (Hematoxylin and eosin,×200).

\section{Supplementary Files}

This is a list of supplementary files associated with this preprint. Click to download.

- CARE.pdf 